UCRL-99555

PREPRINT

\title{
The Development and Characterization of Neutron-Damaged GaAs X-Ray Detectors
}

\author{
P. T. Springer
}

This paper was prepared for submittal to

SPIE's O-E/FIBER LASE Boston '88

Boston, MA, September 6-10, 1988

September 9, 1988
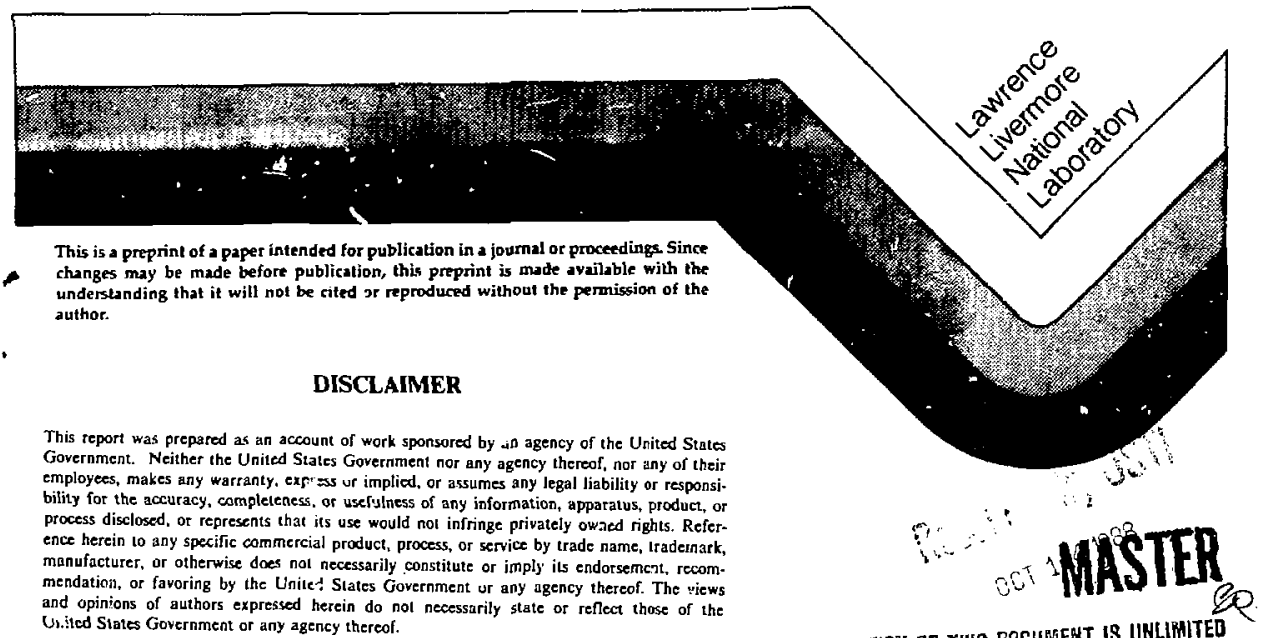
The development and characterization of neutron-damaged GaAs x-ray detectors

\author{
P.T. Springer \\ University of California, Lawrence Livermore National Laboratory \\ P.O. Box 808, Livermore, CA 94551 \\ UCRL- -99555 \\ ABSTRACT DE89 000690
}

Photoconductive $x$-ray detectors are becoming an important $x$-ray diagnostii as a result of their small size, fast response time, and high sensitivity. We are developing a discrete array of neutron-damaged GaAs detectors to be used in an imaging $x$-ray spectrometer, and we describe herein the techniques we use to fabricate and characterize them for an upcoming experiment. Using a 225 -ps $x$-ray pulse from a laser-produced plasma, we measured the sensitivity and time response of the detectors to be $7.1 \mathrm{~mA} / W$ and on the order of $150 \mathrm{ps}$ FWHM, respectively. The carrier mobili; is $741 \mathrm{~cm}^{2} / \mathrm{V} \cdot \mathrm{s}$ at a bias of $2 \times 10^{4} \mathrm{~V} / \mathrm{cm}$.

\title{
1. INTRODUCTION
}

For high-bandwidth detection of photon or charged particle fluxes, photoconductive $x$-ray detectors offer distinct advantages over other detectors. Photoconductive detectors are typically made of semiconductors or insulators - such GaAs, I.AP, and silicon-that can be doped with imprurities or damaged with neutrons or charged particles to reduce the carrier lifetime. As in all semiconductor $x$-ray detectors, radiation entering the detector exciles electrons into the conduction band, giving a current related to the exciting intensity. In other solid-state detectors, such as PIN diodes, the electron hole recombination time is long, and the detector time response is limited by sweepout.

Making detectors thinner not only decreases the sweepout times but also increases the capacitance, which may also limit the response time. In a fast, photoconductive, radiation detector; the response times are determined by carrier recombination rather than sweepout and can be in the sange of a few picoseconds. Thus, the time response of the detectors is somewhat adjustable, with a tradeoff in sensitivity.

Wis are developing an $x$-ray spectrometer that requires a fast and reliable readout. A spherically curved crystal is used to Bragg-reflect and image $x$ rays from severai different sources simultaneously. On the spectrometer output plane, the spectra from the different sources are spaced by only $2 \mathrm{~mm}$, requiring the use of small and finely positioned readout detectors.

The primary means of measuring the $x$-ray intensity is with a streak camera. The $x$ rays deposit their energy in a subnanosecond phosphor of indium-doped CdS, producing a prompi optical signal that is proportional to $x$-ray intensity. The signal is transmitted via a quartz optical fiber to the streak camera. An additional, redundant measurement of the $x$-ray intensity was desired to improve the reliabilicy of the spectrometer.

We chose to use neutron-damaged GaAs detectors because they are small, fast, sensitive, and easy to use. Although only 12 of these detectors will be used in the spectrometer, the ease of microscopic fabrication techniques allowed us to make $200 \mathrm{GaAs}$ chips for use in future experiments.

\section{FABRICATION TECHNIQUES}

Using techniques developed by Ron Wagner and Bob Hammond of Los Alamos National Laboratory (LANL). Mike Pocha of the Energy Research Division of Lawrence Livermore National Laboratory (LLNL) used 5-cmdiameter, 0.5-mm-thick wafers of undoped GaAs to make the detectors, each of which had a $1 \times 1 \times 9.5-\mathrm{mm}$ gap. To produce the ohmic contacts on the detectors, we used microscopic lithographic techniques to deposit $1 \times 1$-mn patterns of layered metal in patterns consisting of $100 \AA$ of gold eutectic (a mixture of $12 \%$ germanium and $88 \%$ gold), $150 \mathrm{~A}$ of nickel. and finally $1000 \mathrm{~A}$ of gold. 
After prolect.ng the patches with photo resist, we etched away the metal between patches and brought the wafer to a temperature of $400^{\circ} \mathrm{C}$ for $10 \mathrm{~s}$ to allow the gold eutectic to alloy with the GaAs. (This is found to produce a good ohmic contact.) We then sawed the wafer into pieces that were slightly larger than $1 \times 1 \mathrm{~mm}$.

Hammond and Wagner then neutron-damaged the GaAs pieces in LA.NL's Omega west reactor. After surrounding the pieces with boson carbide and indium foil to capture thermal neutrons, they placed them near the reactor core for 1000 to $2000 \mathrm{~s}$, producing detectors with response times of 100 to $200 \mathrm{ps}$. (Although v:e have not measured the curve of response time vs neutron dose, it can be said that higher neutron doses result in faster response times but lower sensitivities. With lower neuton doses, the time response is degraded, showing a Jonglived tail. Because a 200-ps response time is adequate and the highest sensitivity is desired, heavy neutrondamaging is not desirable for the $x$-ray spectrometer application.) The chips were allowed to "cool" until they were safe to handle, and then the side into which the $x$ rays enter was polished.

\section{ASSEMBLY, CHECKOUT, AND RESULTS}

The $1 \times 1 \times 0.5-\mathrm{mm}$, neutron-damaged GaAs detectors are mounted in an er:dicap that fits at the end of a 5-cm

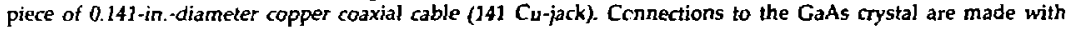
silver epoxy. One side of the crystal is connected, by means of a spring bellows, to the center conductor of the coaxial cable. The other side of the crystal is connected to ground through a protecting endcap. The 141 Cu-jack terminates at an SMA connector.

After assembly, as a check to verify that the detectors function and have adequate time response, we applied a $1-\mathrm{kV}$ bias to them through a high-bandwidth coupling can and excited them with 55-ps pulses of 800-nm light from a GaAs laser diode. Figure 1 shows the measured signal for a detector that had been irradiated for $2000 \mathrm{~s}$. The signal from the detector irradiated for $1000 \mathrm{~s}$ was similar. The detectors show a time response of approximately 150 ps FWHM and exhibit no long-lived tail.

To date, we have calibrated the detectors for sensitivity by using pulsed $x$ rays from a laser-produced plasma. These $x$ rays have a mean energy of $2 \mathrm{keV}$, a pulse duration on the order of $200 \mathrm{ps}$, and a peak intensity of $1.1 \times 10^{4} \mathrm{~W} / \mathrm{cm}^{2}$. We also plan to use dc $\mathbf{x}$-ray sources to calibrate the detectors in order to obtain the comect range of $x$-ray energies of interest in the spectrometer application.

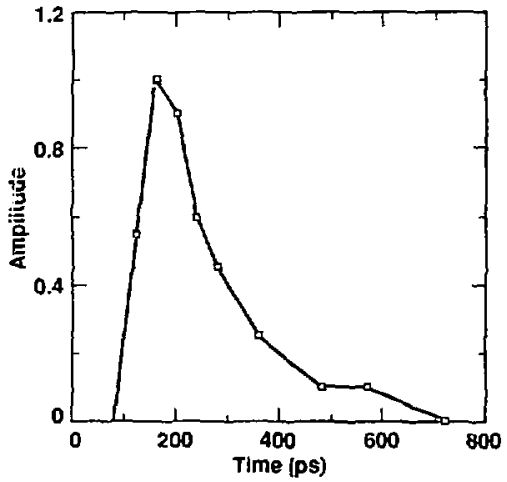

Figure 1. The 150-ps FWHM time response of the photoconductive detector is measured by using a 55 p5 pulse or $820-\mathrm{nm}$ light and a 25 -ps sampiing oscilloscope. The carrier recombination time of $120 \mathrm{ps}$ is de. termined from the exponential falioff of the signal once the exciting pulse stops. 


\subsection{Pulse calibration}

We used the Janus laser facility at LLNL to produce a 200-ps pulse of $x$ rays. The neodymium glass laser was frequency-doubled and operated with a pulse duration of $100 \mathrm{ps}$. We focused the laser to a spot size on the order of $30 \mu \mathrm{m}$ onto a titanium foil. We placed a 127- $\mu \mathrm{m}$ filter of beryllium on a 50-ps, 8201 XRD, and a 50-ps Tektronix 7250 oscilloscope recorded the 225-ps FWHM x-ray pulse (see Fig. 2). The photoconductive detector then measured the $x$-ray pulse, and two silicon PIN diode detectors determined the energy deposition of the $x$-ray pulse.

We placed the photoconductive detector, with a $127-\mu \mathrm{m}$ beryllium $x$-ray filter, $11.4 \mathrm{~cm}$ from the target. The two PIN diodes were each $30.5 \mathrm{~cm}$ from the target and had defining apertures of $2.17 \mathrm{~mm}^{2}$. One of the PIN diodes, with a filter identical to that of the photoconductive detector, measured the $x$-ray intensity behind $127 \mu \mathrm{m}$ of heryllium. The other PIN diode, with an x-ray filter of $50.8 \mu \mathrm{m}$ of beryllium, determined the mean energy of the $x$ rays.

Figures 3, 4, and 5 show the oscilloscope traces from a 2.64-J laser shot, as measured by the photoconductive detector and two PIN diode detectors. The photoconductive signal has an FWHM of $475 \mathrm{ps}$, which is close to the bandwidth limit of the Tektronix 7104 oscilloscope used to record the signal. We used the oscilloscope to record a 60-ps pulse from a pulse generator, and the trace is shown in Fig. 6 . The obsezved FWHM of the trace shown in Fig. 6 implies an oscilloscope response time on the order of $400 \mathrm{ps}$. The 475 -ps width of the observed photoconductive signal is a combination of the photoconductive response time, the 225-ps time duration of the $x$-ray pulse, and the 400 -ps oscilloscope response time. The observed trace is consistent with a photoconductive time response on the order of $150 \mathrm{ps}$, as was seen using the GaAs laser diode.

The signals shown in Figs. 3 and 4 are used to determine the sensitivity of the photoconductive detectors. The PIN diodes were calibrated and shown to have a flat response. The sensitivity of the PIN diodes is $0.2 \mathrm{C} / \mathrm{I}$. The signal shown in Fig. 4 shows $1.84 \mathrm{nC}$ of charge collected, implying a total of $293 \mu \mathrm{J} / \mathrm{sr}$ of $\mathrm{x}$ rays behind the $127-\mu \mathrm{m}$ beryllium filter for the $2.64 \mathrm{~J}$ laser shot. Integrating the photoconductive signal gives $80 \mathrm{pC}$ of charge for the $11.2 \mathrm{n}$ ] of incident $x$ rays. This implies an efficiency of $7.1 \mathrm{mC} /$ ].

The theoretical efficiency of the photoconductive detector is given by

$\eta_{\mathrm{pdd}}=e v \tau / w E_{\mathrm{eh}}$.

Here, $e$ is the electron charge, $v$ is the carrier drift velocity, $z$ is the carrier recombination time, $w=0.5 \mathrm{~mm}$ is the gap width, and $E_{\mathrm{wh}}=5 \mathrm{eV}$ is the energy per electron-hole pair in GaAs. The measured efficiency implies a value of

Fig'ıre 2. The lanus laser focused onto a titanium target produces a 225 -ps $x$-ray pulsc, as measured by a 50-ps $8201 \times$-ray diode and recorded by a 50-ps Tektronix 7250 oscilloscope.

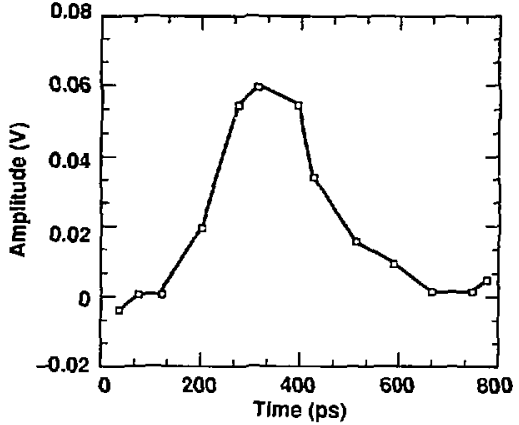




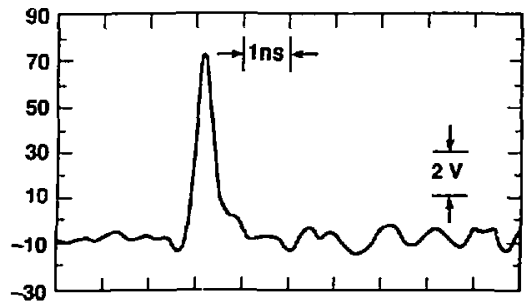

Figure 3. The PCD signal from the Janus $x$-ray pulse, as recorded by a Tektronix 7104 oscilloscope.

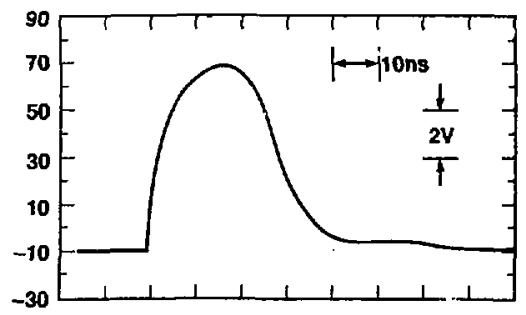

Figure 5. Another PIN diode is used to measure the total energy in the $x$-ray pulse that passes through a $51-\mu \mathrm{m}$ beryllium filter. The PIN diode gives $4.06 \mathrm{nC}$ of charge. Using the detector sensitivity and solid angle implies a filtered $x$-ray pulse strength of $648 \mu \mathrm{l} / \mathrm{sr}$ for the 2.64-J laser shot. Based on the differential attenuation, the mean x-ray energy is about $2 \mathrm{keV}$.

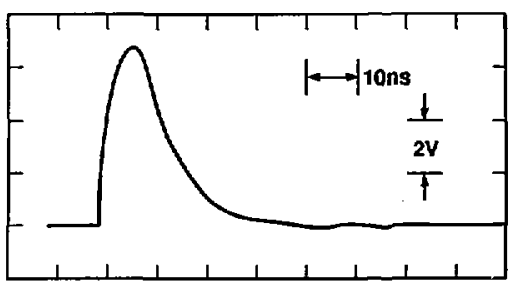

Figure 4. A PIN diode is used to measure the rytal energy in the $x$-ray pulse that passes through a $127-\mu \mathrm{m}$ beryllium filter. The PIN diode gives $1.84 \mathrm{nC}$ of charge. Using the detector sensitivity and solid angle implies a filtered $x$-ray pulse strength of $294 \mu \mathrm{J} / \mathrm{sr}$ for the 2.64-] lasser shot.

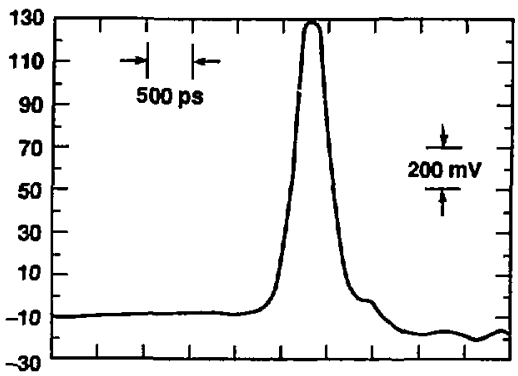

Figure 6. The Tektronix 7104 oscilloscope response time, as measured by a 60 -ps pulser, is 400 os FiHHM.

$17.8 \mu \mathrm{m}$ for the product $v \tau$. The detector has only $3.6 \%$ of the intrinsic efficiency because of the neutronJamaging. With an estimated $1 / e$ recombination time of $120 \mathrm{ps}$, the drift velocity is on the order of $1.48 \times 10^{7} \mathrm{~cm} / \mathrm{s}$. This value is below that of intrinsic $\mathrm{GaAs}$ biased at $20,000 \mathrm{~V} / \mathrm{cm}$. The carrier mobiiity, $\mu$, is $741 \mathrm{~cm}^{2} / \mathrm{V} \cdot s$ at this bias level.

\subsection{De calibration}

The second method for calibrating the detectors is by the lise of $x$ rays from standard $x$-ray tubes. When biased, thie GaAs detectors have a leakage current of about $1 \mu \mathrm{A}$, which is caused by the resistance of the GaAs. This leakage curren: tar exceeds the typical currents induced by laboratory $x$-ray tubes. To measure the smallest $x$-ray induced currents, we balance the leakage current of one photoconductive detector against that of another that is bizsed with a voltage of the opposite polarity. The leakage currents are combined and measured with a 
sensitive electrometer. The bias voltage of the compensating PCD is adjusted to zero the reading when no $x$ rays are applied. Differential leakage currents as low as $1 \mathrm{nA}$ are obtained. The stability of the differential leakage current is about $1 \mathrm{nA} / \mathrm{s}$, which is sufficient to allow a determination of the $x$-ray-induced current. At the time of this writing, however, these calibrations have not yet been performed.

\section{ACKNOWLEDGMENTS}

I would like to thank Mike Pocha for his heip in the detector fabrication and Ron Wagner and Bob Hammond for their help in the detector irradiations. 1 thank George Wells for his help in the detector assembly. Russell Benjamin operated the $x$-ray diode and fast ocscilloscope for the Janus run.

This work was performed under the auspices of the U.S. Department of Energy by the Lawrence Livermore National Laboratory under contract No. W-7405-Eng-48. 